\title{
Lectin-like, oxidized low-density lipoprotein receptor-1-deficient mice show resistance to age-related knee osteoarthritis: A Mini review Kazuhiko Hashimoto $^{1 *}$, Yutaka Oda ${ }^{1}$, Shigeshi Mori ${ }^{2}$, Koutaro Yamagishi ${ }^{1}$, Tsukamoto Ichiro ${ }^{1}$, Masao Akagi 'Department of Orthopedic Surgery, Kindai University Hospital, Osaka-Sayama City, Osaka 589-851 1, Japan ${ }^{2}$ Department of Orthopedic Surgery, Kindai University Nara Hospital, Ikoma City, Nara 630-0293, Japan
}

Article Info

\section{Article Notes}

Received: May 31, 2018

Accepted: June 26, 2018

\section{*Correspondence:}

Dr. Kazuhiko Hashimoto, Department of Orthopedic Surgery, Kindai University Hospital, 377-2 Ohno-Higashi, Osaka-Sayama City, Osaka 589-8511, Japan; Telephone No: +81-072-366-0221; Fax No: +81-072-366-0206; Email: hazzhiko@med.kindai.ac.jp

(c) 2018 Hashimoto K. This article is distributed under the terms of the Creative Commons Attribution 4.0 International License.

\section{Keywords}

Osteoarthritis (OA)

LOX-1

Low-density lipoprotein

MMP-13

COL-X

Cartilage degeneration

Knockout

Immunohistochemistry

\section{ABSTRACT}

The lectin-like, oxidized low-density lipoprotein (ox-LDL) receptor-1 (LOX1)/ox-LDL system contributes to atherosclerosis and thus may play a role in cartilage degeneration. The purpose of this study was to determine whether the LOX-1/ox-LDL system contributes to the pathogenesis of age-related osteoarthritis (OA) in vivo, using LOX-1 knockout (LOX-1 KO) mice. Knee cartilage samples from 6-, 12-, and 18-month-old ( $\mathrm{n}=10$ per group) $\mathrm{C57BI} / 6$ wild-type (WT) and LOX-1 KO mice were compared for OA-related changes with Safranin-O staining. At 12 and 18 months, the OA changes were significantly reduced in LOX-1 KO mice compared to those in WT mice. Moreover, an immunohistological analysis showed that the expression levels of Runt-related transcription factor-2, type- $X$ collagen, and matrix metalloproteinase-13 in the articular chondrocytes were significantly decreased in LOX-1 KO mice compared with those in WT mice. Overall, this study indicates that the LOX-1/ ox-LDL system in chondrocytes plays a role in the pathogenesis of age-related knee OA, highlighting a novel potential target for preventing OA progression.

\section{Introduction}

Oxidized low-density lipoprotein (ox- LDL) is produced by LDL oxidation at sites of oxidative stress and inflammation, and thus, plays an important role in the pathogenesis of atherosclerosis ${ }^{1}$. Lectin-like ox-LDL receptor-1 (LOX-1) is an important receptor for ox-LDL, which was originally cloned from cultured bovine aortic endothelial cells ${ }^{2}$. LOX-1 has also been widely shown to participate in degeneration of the articular cartilage both in vivo and in vitro ${ }^{3-12}$. However, the influence of LOX-1 on the age-related progression of $\mathrm{OA}$ in vivo remains unclear. In particular, we previously reported that the LOX-1/ox-LDL system induces chondrocyte hypertrophy in vitro via Runt-related transcription factor-2 (Runx2) expression, and that LOX-1 knockdown reduced Runx2 expression ${ }^{10}$. These previous findings led us to hypothesize that the LOX-1/ox- LDL system is involved in age-related cartilage degeneration via chondrocyte hypertrophy in vivo. Therefore, in the present study, we conducted histological observations of $\mathrm{OA}$ development in the articular cartilages of wild-type (WT) and LOX-1 knockout (KO) mice, which were maintained for up to 18 months of age.

\section{Material and Methods}

\section{Mice}

LOX-1 $1^{+/+}$C57BL/6 Jcl mice (WT) were provided by Nihon CLEA (Tokyo, Japan). The LOX-1/- C57BL/6 Jcl mice (KO) were originally generated by Sawamura et al. ${ }^{13}$ and provided by the National Cerebral 
and Cardiovascular Center (Osaka, Japan). Mice were housed in cages with access to food and water ad libitum in a temperature-controlled room with a 12-h dark/12-h light cycle. All animal experiments were performed in accordance with protocols approved by the Animal Care and Use Committee of our hospital.

\section{Safranin O staining and immunohistochemistry}

To monitor age-related $\mathrm{OA}$, articular cartilage samples of WT and LOX-1 KO mice were examined at 6, 12, and 18 months of age ( $n=10$, in each). We also recorded the body weights of the mice at the same three time points.

The knee samples were stained with Safranin 0 (WAKO, Japan) for histological evaluation of cartilage degeneration during OA progression at 6,12 , and 18 months of age ${ }^{2}$. Knee $\mathrm{OA}$ was evaluated using the OARSI scoring system which is a semi-quantitative scoring system ${ }^{14}$. We also divided the prevalence of OA by the OARSI score in both WT and LOX-1 KO mice at each time point.

To visualize LOX-1 and ox-LDL expression and investigate the involvement of cartilage cell hypertrophy in OA progression, we performed immunohistochemistry against LOX-1, ox-LDL, Runx2, and type-X collagen (COL $\mathrm{X})$. Immunohistochemistry was also used to detect matrix metalloproteinase-13 (MMP-13), which is a cartilage matrix-degrading enzyme.

\section{Statistical analysis}

All data are expressed as the mean \pm standard deviation. The scores of each group were compared using Student's t-test. P-values of less than 0.05 were considered statistically significant.

\section{Results}

\section{OA development}

At 6 months age, there was no significant difference between the OA changes of WT and LOX-1 KO mice. However, at 12 and 18 months, there were significant differences between OA changes of the two groups, and LOX-1 KO mice showed significantly reduced scores reflecting OA than WT mice. The mean body weights were not significantly different between WT and LOX-1 KO mice at any time point tested.

\section{Prevalence of $\mathrm{OA}$}

The prevalence of OA in LOX-1 KO mice was lower than that in WT mice at 12 and 18 months of age (40\% vs. $70 \%$, and $70 \%$ vs. $90 \%$, respectively; $n=10$ ).

\section{Time course of LOX-1, ox-LDL, Runx2, COL X, and MMP-13 expression in the cartilage}

In the WT mice, the staining intensity for LOX-1 and ox-
LDL increased at 12 and 18 months of age, compared to that detected at 6 months. However, no LOX-1 or ox-LDL staining was observed in articular cartilage sections of LOX1 KO mice. Similar results were obtained for Runx2, COLX, and MMP-13 immunohistochemical staining at 6 months in the two groups. However, at 12 and 18 months, LOX-1, oxLDL, Runx2, and MMP-13 expression was increased in both groups of mice, although significantly lower expression was observed in LOX-1 KO mice.

\section{Discussion}

We demonstrated that the loss of LOX-1 prevented the progression of age-related cartilage degeneration in the murine knee. Furthermore, LOX-1/ox-LDL expression increased with OA progression in WT mice. These findings suggest that LOX-1 plays an important role in cartilage degeneration during age-related $\mathrm{OA}$ progression in vivo.

Chondrocyte senescence is known to drive the development of age-related $\mathrm{OA}^{15,16}$. Interestingly, telomere shortening has also been detected in chondrocytes isolated from the articular cartilage of older adults ${ }^{17}$. We previously reported that ox-LDL binding to LOX-1 promotes stressinduced premature senescence in chondrocytes, resulting in suppressed telomerase activity ${ }^{9}$. Furthermore, oxidative changes are important for chondrocyte senescence in cartilage degeneration ${ }^{18,19}$. The ox-LDL-LOX-1 interaction induces reactive oxygen species (ROS) production in bovine articular chondrocytes ${ }^{8}$.

Recent studies have indicated that endochondral ossification signals, which cause hypertrophy and apoptosis in chondrocytes, are involved in age-related OA development ${ }^{20,21}$. A hypertrophic phenotype was also observed in an age-related OA mouse model and in human OA chondrocytes ${ }^{22,23}$. We previously reported that ox-LDLLOX-1 binding induces ROS production ${ }^{8}$, and ROS were recently shown to induce chondrocyte hypertrophy ${ }^{24}$. It is also well established that cartilage degeneration involves various enzymes, including MMPs ${ }^{25,26}$. Specifically, MMP-13 is a major cartilage degradation enzyme that contributes to OA progression ${ }^{27,28}$, particularly for that occurring in agerelated $\mathrm{OA}^{29,30}$.

Our present results expand on these known mechanisms by pointing to a novel role of the LOX-1/ox-LDL system in cartilage degeneration via mediating MMP-13 expression in vivo. Since LOX-1 deficiency suppressed OA development in a murine model of age-related $\mathrm{OA}$, addressing or treating atherosclerosis may help to prevent OA.

\section{Acknowledgements}

We thank to Editage for English editing.

\section{Conflicts of interest}

The authors have no conflict of interest to declare. 


\section{References}

1. Zhang PY, Xu X, Li XC. Cardiovascular diseases: oxidative damage and antioxidant protection. Eur Rev Med Pharmacol Sci. 2014; 18: 3091-6.

2. Sawamura T, Kume N, Aoyama T, et al. An endothelial receptor for oxidized low-density lipoprotein. Nature. 1997; 386: 73-7.

3. Nakagawa T, Akagi M, Hoshikawa H, et al. Lectin-like oxidized lowdensity lipoprotein receptor 1 mediates leukocyte infiltration and articular cartilage destruction in rat zymosan-induced arthritis. Arthritis Rheum. 2002; 46: 2486-94.

4. Akagi M, Kanata S, Mori S, et al. Possible involvement of the oxidized low-density lipoprotein/lectin-like oxidized low-density lipoprotein receptor-1 system in pathogenesis and progression of human osteoarthritis. Osteoarthr Cartilage. 2007; 15: 281-90.

5. Nakagawa T, Yasuda T, Hoshikawa H, et al. LOX-1 expressed in cultured rat chondrocytes mediates oxidized LDL-induced cell death-possible role of dephosphorylation of Akt. Biochem Biophys Res Commun. 2002; 299: 91-7.

6. Kakinuma T, Yasuda T, Nakagawa T, et al. Lectin-like oxidized lowdensity lipoprotein receptor 1 mediates matrix metalloproteinase 3 synthesis enhanced by oxidized low-density lipoprotein in rheumatoid arthritis cartilage. Arthritis Rheum. 2004; 50: 3495-503.

7. Simopoulou T, Malizos KN, Tsezou A. Lectin-like oxidized lowdensity lipoprotein receptor 1 (LOX-1) expression in human articular chondrocytes. Clin Exp Rheumatol. 2007; 25: 605-12.

8. Nishimura S, Akagi $M$, Yoshida $K$, et al. Oxidized low-density lipoprotein (ox-LDL) binding to lectin-like ox- LDL receptor 1 (LOX1) in cultured bovine articular chondrocytes increases production of intracellular reactive oxygen species (ROS) resulting in the activation of NF-кB. Osteoarthr Cartilage. 2004; 12: 568-76.

9. Zushi $\mathrm{S}$, Akagi $\mathrm{M}$, Kishimoto $\mathrm{H}$, et al. Induction of bovine articular chondrocyte senescence with oxidized low-density lipoprotein through lectin-like oxidized low-density lipoprotein receptor 1 . Arthritis Rheum. 2009; 60: 3007-16.

10. Kishimoto H, Akagi M, Zushi S, et al. Induction of hypertrophic chondrocyte- like phenotypes by oxidized LDL in cultured bovine articular chondrocytes through increase in oxidative stress. Osteoarthr Cartilage. 2010; 18: 1284-90.

11. Hashimoto K, Mori S, Oda Y, et al. Lectin-like oxidized low density lipoprotein receptor 1-deficient mice show resistance to instabilityinduced osteoarthritis. Scand J Rheumat. 2016; 45: 412-22.

12. Hashimoto $\mathrm{K}$, Oda $\mathrm{Y}$, Nakagawa $\mathrm{K}$, et al. LOX-1 deficient mice show resistance to zymosan-induced arthritis. Eur J Histochem. 2018; 62(1): 2847

13. Mehta JL, Sanada N, Hu CP, et al. Deletion of LOX-1 reduces atherogenesis in LDLR knockout mice fed high cholesterol diet. Circ Res. 2007; 100: 1634-42.

14. Glasson SS, Chambers MG, Van Den Berg WB, et al. The OARSI histopathology initiative - recommendations for histological assessments of osteoarthritis in the mouse. Osteoarthr Cartilage. 2010; 18: S17-23.

15. Hui W, Young DA, Rowan AD, et al. Oxidative changes and signalling pathways are pivotal in initiating age-related changes in articular cartilage. Ann Rheum Dis. 2016; 75: 449-58.

16. Anderson AS, Loeser RF. Why is osteoarthritis an age-related disease. Best Pract Res Clin Rheumatol. 2010; 24: 15-26.

17. Kume N, Murase T, Moriwaki H, et al. Inducible expression of lectinlike oxidized LDL receptor-1 in vascular endothelial cells. Circ Res. 1998; 83: 322-7.

18. Hui W, Young DA, Rowan AD, et al. Oxidative changes and signalling pathways are pivotal in initiating age-related changes in articular cartilage. Ann Rheum Dis. 2016; 75: 449-58.

19. Lepetsos P, Papavassiliou AG. ROS/oxidative stress signaling in osteoarthritis. Biochim Biophys Acta. 2016; 1862: 576-91.

20. Baugé C, Girard N, Lhuissier E, et al. Regulation and role of TGF $\beta$ signaling pathway in aging and osteoarthritis joints. Aging Dis. 2013; 5: 394-405.

21. Fujita N, Matsushita T, Ishida K, et al. Potential involvement of SIRT1 in the pathogenesis of osteoarthritis through the modulation of chondrocyte gene expressions. J Orthop Res. 2011; 29: 511-5.

22. Ailixiding M, Aibibula Z, Iwata M, et al. Pivotal role of Sirt6 in the crosstalk among ageing, metabolic syndrome and osteoarthritis. Biochem Biophys Res Commun. 2015; 466: 319-26.

23. Aigner T, Reichenberger E, Bertling W, et al. Type X collagen expression in osteoarthritic and rheumatoid articular cartilage. Virchows Arch B Cell Pathol Incl Mol Pathol. 1993; 63: 205-11.

24. Morita K, Miyamoto T, Fujita N, et al. Reactive oxygen species induce chondrocyte hypertrophy in endochondral ossification. J Exp Med. 2007; 204: 1613-23.

25. Cawston TE, Wilson AJ. Understanding the role of tissue degrading enzymes and their inhibitors in development and disease. Best Pract Res Clin Rheumatol. 2006; 20: 983-1002.

26. Burrage PS, Mix KS, Brinckerhoff CE. Matrix metalloproteinases: role in arthritis. Front Biosci. 2006; 11: 529-43.

27. van den Berg WB. Osteoarthritis year 2010 in review: pathomechanisms. Osteoarthr Cartilage. 2011; 19: 338-41.

28. Mitchell PG, Magna HA, Reeves LM, et al. Cloning, expression, and type II collagenolytic activity of matrix metalloproteinase-13 from human osteoarthritic cartilage. J Clin Invest. 1996; 97: 761-8.

29. Blaney Davidson EN, Remst DF, Vitters EL, et al. Increase in ALK1/ ALK5 ratio as a cause for elevated MMP-13 expression in osteoarthritis in humans and mice. J Immunol. 2009; 182: 7937-45.

30. Weng T, Xie Y, Yi L, et al. Loss of Vhl in cartilage accelerated the progression of age-associated and surgically induced murine osteoarthritis. Osteoarthr Cartilage. 2014; 22: 1197-205. 